\section{Biodiversity in model ecosystems}

SIR - In his News and Views article, Kareiva $^{1}$ applauded the innovative approach of Naeem et al. ${ }^{2}$, who carried out experiments on ecosystems under controlled and duplicable conditions. Kareiva is of the view that field experiments on ecosystems will always be necessary, but they tend to be hard to repeat. This has often been a stumbling block in ecology research. Experiments on small microcosms do not suffer from this difficulty.

One criticism of microcosm-based research is that enclosure in a small volume is somehow 'unnatural'. But our terrestrial biosphere, the best natural example, is essentially a closed system. Enclosure (not necessarily full closure) paves the way to an experimental approach in ecology that is more receptive to advanced instrumentation, and consequently offers a better control over experimental conditions, and allows quantification of the observations and a powerful exploration of their validity by means of comparison and repetition.

In the experiment designed by Naeem $e t$ $a l .^{2}$, the productivity of three ecosystem communities (their ability to produce biomass) is shown to rank parallel to their biodiversity. None of the other ecosystem processes measured (decomposition, nutrient retention and water retention) exhibited a consistent pattern of variation among treatments. We believe that the choice of plant species in these three communities is not entirely justified in that it does not appear to have been motivated by a concern about biodiversity loss. We question the selection of Senecio vulgaris and Stellaria media in the poorest community instead of, for example, larger species in the pool considered. Also, there is a mismatch between the size of the large plants (about $60 \mathrm{~cm}$ ) and the small size of the soil samples $(1 \mathrm{~m} \times 1 \mathrm{~m})$ to be colonized. Attempts to balance the three communities based on the initial number of seeds ( 80 seeds for each community) is a necessary, but perhaps not sufficient, precaution. In particular, large plants (taller than $50 \mathrm{~cm}$ ) were present only in the medium- and high-diversity communities whereas the low-diversity community consisted only of small plants (smaller than 30 $\mathrm{cm}$ ). It is well known that a monospecific culture can be as productive as a mixed culture, provided that the species has been selected, or adapted, to exhibit this potential. Another choice for the first community, featuring a large plant species with a canopy structure capable of optimizing the three-dimensional colonization of the available space, might have yielded a distinct ranking.

We appreciate that the purpose of the study of Naeem et al. was not to identify the surviving species in an environment where biodiversity is declining, but we wish to draw attention to the difficult problem of adequately 'selecting' species to simulate a low-diversity community. Provided special attention is devoted to this problem, similar experimental approaches, in which producerconsumer-decomposer interactions in controlled environments are integrated, offer a new and promising context for studying biodiversity in liaison to ecosystem stability. The study of Naeem et al. is a pioneering experiment in pointing the way in this direction.

\section{Marcel André}

François Bréchignac

Pierre Thibault

CEA-Direction des Sciences du Vivant,

Département Physiologie Végétale et

Ecosystèmes,

Centre d'Etudes de Cadarache,

BP1, 13108-Saint Paul lez Durance Cedex, France

NAEEM ET AL. REPLY - André et al. are correct to say that the choice of species in a manipulation of diversity critically determines its outcome. They suggest that exclusion of a single plant species, Chenopodium album, from the low-diversity treatment in our experiment ${ }^{2}$, may have been solely responsible for the positive association between biodiversity and ecosystem productivity. Species diversity is a difficult variable to manipulate experimentally because of the many possible species combinations such manipulations can generate. We were aware of the importance of the problem and took the following three steps to ensure that our experiment would not be overly sensitive to the presence or absence of one or a few species.

(1) Design. We used relatively ecologically homogeneous species within trophic classes to ensure against the possibility of 'keystone' species effects ${ }^{1,2}$.

(2) Calibration of the Ecotron plant communities. A glass-house experiment (S. N. et al., submitted; see figure) compared the potential productivity of the plant species combinations grown in the Ecotron with the productivities of alternative combinations. Results support findings in the Ecotron in two ways. First, the relationship between productivity and species richness (black bars) is positive. Second, the specific combinations used in the Ecotron (white bars and the black 16-species bar) were equal in potential productivity (ANOVA; d.f. $=2,25 ; F=$ $0.23 ; P=0.79$ ). Thus, the combinations of species used in the Ecotron provided a conservative test of the association between diversity and productivity.

(3) Keystone species effects. C. album, although tall, was not a keystone species. Under glass-house conditions, C. album is

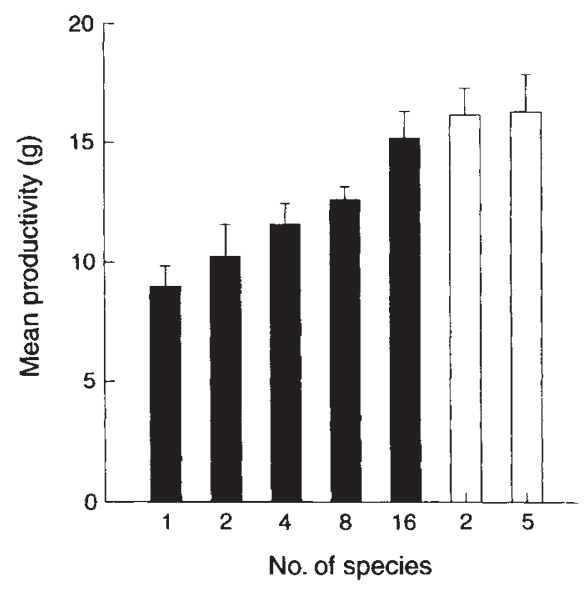

Calibration of the productivity of Ecotron plant species combinations against other possible combinations (S. N. et al., submitted). Black bars are means of above-ground plant biomass (dry weight \pm 1 s.e.) from pots containing 16 individual plants that ranged in diversity between 1 (monocultures), 2, 4, 8, (intermediate richness combinations) and 16 species (full richness combinations) from the pool of 16 plant species used in the Ecotron experiment ${ }^{2}$. Intermediate richness combinations were random selections of species, without duplication, drawn from the pool of 16. White bars, the specific 2-species and 5-species combinations used in the Ecotron. The number of replicates were 4 for each monoculture and 10 for full richness combinations. Intermediate richness combinations consisted of 19,30 and 40 unique combinations of 2, 4 and 8 species, respectively. Ecotron combinations of 2 species and 5 species had 10 and 8 replicates, respectively. Soil was identical to soil used in the Ecotron. Plants were grown between 10 May and 16 July 1993 (one plant generation) in a glass house at Silwood Park.

not the most productive species, ranking sixth in productivity; Senecio vulgaris and Stellaria media, the plant species universal to all treatments, ranked second and third highest in productivity. Second, C. album accounted for $20.4 \%$ of sampling pin encounters with vegetation in intermediate- and $8.36 \%$ of encounters in highdiversity mesocosms. These measured abundances accord with expected encounters of $20.0 \%$ ( $1 / 5$ of species) and $6.25 \%$ ( $1 / 16$ of species) for intermediate- and high-diversity mesocosms, respectively.

Shahid Naeem*

Lindsey J. Thompson

Sharon P. Lawler*

John H. Lawton

Richard M. Woodfin

NERC Centre for Population Biology,

Imperial College at Silwood Park,

Ascot, Berks SL5 7PY, UK

*Present addresses: Department of Ecology, Evolution and Behaviour, University of Minnesota. St Paul. Minnesota 55108, USA (S.N.); Morgan School of Biological Sciences, University of Kentucky, Lexington, Kentucky 40506, USA (S.P.L.).

1. Kareiva, P. Nature 368, 686-687 (1994)

2. Naeem, S. etal. Nature 368, 734-737 (1994) 